\title{
ROP surgery and ocular circulation
}

WAJ van Heuven and JW Kiel

surgeons have abandoned vitreoretinal surgery for ROP. This poses the question whether the surgery and/or the post-operative care cause the severe optic atrophy often observed later. This report analyses the results of ROP surgery performed by experienced surgeons in the United States, the techniques used as well as data about blood pressure (BP) and intraocular pressure (IOP) in premature infants. A hypothesis is proposed that ocular circulation during or after surgery may be compromised. An underlying premise of this hypothesis is that the ocular perfusion pressure (OPP) is defined as the mean arterial pressure (MAP) minus the IOP and that chorioretinal blood flow ceases when the difference between MAP and IOP approaches zero. Previous experiments in anaesthetized rabbits, where choroidal blood flow was measured at different fixed levels of MAP as the IOP was increased, clearly demonstrate that the MAP minus the IOP is the effective OPP and that choroidal blood flow ceases when the OPP becomes zero. ${ }^{2}$

\section{Materials and Methods}

Published surgical results were reviewed. Experienced ROP surgeons were asked about their techniques, the instruments utilized, IOP and BP during surgery, intraocular vitreous substitutes, length of surgery, and complications. Ophthalmic, paediatric, and anaesthesia literature concerning BP in premature infants in the nursery and operating room were reviewed. BP measurements in NICU infants $(n=19)$ were also tabulated.

Keywords: retinopathy of prematurity; ocular blood flow; ocular perfusion pressure; intraocular pressure; vitrectomy

\section{Introduction}

Visual results following vitreoretinal surgery for stages 4 and 5 retinopathy of prematurity (ROP) are frequently disappointing, even when anatomic results are good. ${ }^{1}$ As a result, some
Results

Surgical results

Clinical studies have demonstrated that peripheral retinal ablation can improve structural and functional outcomes in children with severe ROP.,4 However, progression to retinal detachment (RD) still occurs in some infants. These RDs are divided into partial RD
Department of Ophthalmology, University of Texas Health Science Center at San Antonio, San Antonio, TX, USA

Correspondence: JW Kiel, Department of Ophthalmology, University of Texas Health Science Center at San Antonio,

7703 Floyd Curl Drive, San Antonio, TX 78229 3900, USA

Tel: + 12105678474 ; Fax: + 12105678413

E-mail: kiel@

uthscsa.edu

Received: 30 October 2007 Accepted in revised form: 10 January 2008 Published online: 21 March 2008 
(stage 4) and total RD (stage 5).,5 Stage $4 \mathrm{~A}$ is a partial RD not involving the macula and stage $4 \mathrm{~B}$ is a partial $\mathrm{RD}$ with detachment of the macula. In all these stages the results without surgery are generally poor, although an occasional eye with stage $4 \mathrm{~A}$ stabilizes. ${ }^{7}$ Scleral buckling and vitrectomy procedures can successfully re-attach the retina in all stages but the functional results of this surgery are poor. ${ }^{4}$

A recent publication analyses the data from the Early Treatment for Retinopathy of Prematurity study (ETROP). ${ }^{7}$ RD occurred in 89 eyes of 63 patients. Because not all infants were followed and some patients died, the outcomes were tabulated for 66 eyes of 52 patients who underwent surgery and 12 eyes of 11 patients who were not operated. The type of surgery performed was left to the discretion of the surgeon, so that scleral buckling, vitrectomy, or both were done without any specific protocol. In stage $4 \mathrm{~A}$, scleral buckling alone was better than observation, with retinal reattachment in five out of six and some visual acuity in six out of six, with excellent vision in three out of six. Following vitrectomy for stage $4 \mathrm{~A}$, macular attachment remained in 5 out of 18 with some retina attached in 10 out of 18 . However, there was some vision in only six out of eighteen, with good vision in only four. In stage $4 \mathrm{~B}$, most surgeons performed vitrectomy, which resulted in five of ten having total or macular retinal reattachment with four additional partial reattachments. However, there were only two eyes with measurable vision. Vitrectomy for stage 5 resulted in two of eleven partially and two totally reattached retinas, but only two eyes with poor vision and the others with essentially no vision.

\section{Surgical techniques}

Among the surgeons interviewed who performed scleral buckling there was some uniformity of procedure, which lasted from 30 to $90 \mathrm{~min}$, with anaesthesia time about 30 min longer. At times subretinal fluid was drained, making it easier to create a tighter/higher scleral buckle without increasing the IOP excessively. All surgeons regularly observed optic nerve perfusion to assure that the central retinal artery was not occluded. Intraocular air or gas was not used.

Vitrectomies were done with or without a scleral buckling procedure. Two or three ports of entry into the eye were placed $1 \mathrm{~mm}$ posterior to the limbus to avoid the lens. The ports gave access for infusion, suction, manipulation, cutting, and illumination. Most surgeons used $30 \mathrm{~mm} \mathrm{Hg}$ infusion pressure during surgery, although some stated that they used the lowest infusion pressure that did not collapse the eye. Others stated that they frequently looked at the optic nerve retinal vessels to assess blood flow. Intraocular vitreous substitutes were used in most cases with room air most commonly, SF6 and C3F8 less commonly and Healon or oil rarely. Instrumentation ranged from 25 to 18 gauge. The specific surgical and anaesthesia records were not reviewed due to difficulties related to medical record access.

\section{Post-operative complications and management}

Postoperatively most infants were admitted to the hospital until medically stable in 1-7 days. Eye examinations were done daily. Corneal clouding was usually not observed. Retinal vessels appeared perfused. Retinal reattachment was observed in many eyes. IOP measurements were generally not done. Examining ophthalmologists were generally unaware of BP during and after hospital discharge. After discharge, infants were seen irregularly, because many had been referred and were unavailable to be examined by the surgeon.

\section{Anaesthesia during ROP surgery}

Anaesthesia was done using a variety of anaesthetic agents. We found no published data about anaesthesiology in ROP vitrectomy surgery, but there are data suggesting that anaesthesia that ablates all stress responses decreases mortality. ${ }^{8}$ Generally, a 20-30\% drop in BP following induction was common. During anaesthesia the patients were supine and BP was taken using a sphygmomanometer cuff.

For BP during surgery, anaesthesiologists varied in their responses, but all indicated awareness that very low BP can occur, and that they take 'appropriate measures' to keep BP 'normal'. No anaesthesiologists were aware of the IOP during surgery or the issue of ocular perfusion rather than just tissue perfusion. Little discussion took place during surgery between anaesthesiologist and surgeon concerning OPP.

\section{Blood pressure in the newborn}

Until recently few data existed concerning normal BP in neonates, and there is also uncertainty about the level of $\mathrm{BP}$ required to maintain oxygen delivery to vital organs such as brain and retina. The Brompton Study reported that systolic BP was $76 \mathrm{~mm} \mathrm{Hg}$ in normal neonates (< age 4 days) when awake, and $6 \mathrm{~mm} \mathrm{Hg}$ lower when asleep. ${ }^{9}$ It emphasized the use of an appropriately sized cuff for accurate measurements. Variability of BP measurements taken at different times was large and ranged from 10 to $14 \mathrm{~mm} \mathrm{Hg}$. Thus, a standard protocol for BP measurement in the newborn was recommended by Nwankwo et $a l^{10}$ to minimize variability related to feeding, hunger, and the degree of a baby's restful state. 
A relevant article by Victor et al ${ }^{11}$ looked at BP measured directly in very low birth weight newborns. The mean gestational age was the same as in the ETROP study (27 weeks). The study measured BP with arterial catheters, cerebral electrical activity, fractional oxygen extraction, and peripheral blood flow. The median (range) of MAP was $32 \mathrm{~mm} \mathrm{Hg}$ (16-46). The results also indicated that cerebral perfusion is maintained at an MAP above $23 \mathrm{~mm} \mathrm{Hg}$. In addition, the peripheral perfusion diminishes at MAP levels above the critical level of MAP where cerebral electrical activity becomes abnormal, and cerebral oxygen delivery is maintained at MAP levels between 23 and $33 \mathrm{~mm} \mathrm{Hg}$, probably by diminished peripheral perfusion.

Other recent studies emphasize the cardiovascular instability in the first few weeks of birth in infants less than $1000 \mathrm{~g}$ and less than 28 weeks of gestation. ${ }^{12,13}$ Apparently the pre-term neonate is poorly equipped to handle the cardiovascular changes during the perinatal period, especially if illnesses are superimposed. For example, a patent ductus arteriosus may not close for many days, which can result in left-to-right shunting, with a systemic steal diastolic hypotension. ${ }^{13}$ The general recommendation thus seems to be that BP needs to be monitored frequently and that, should the MAP go below $30 \mathrm{~mm} \mathrm{Hg}$, medical support should be instituted (eg dopamine). ${ }^{14}$

\section{Snapshot of babies in NICU at University Hospital San Antonio, TX}

The infants were evaluated $(n=19)$ on a random day in 2007 and included all infants in the NICU. Mean gestational age at birth was $214 \pm 38$ days (mean $\pm \mathrm{SD}$ ). Figure 1 shows that MAP and body weight correlated linearly with gestational age at the time of measurement ( $247 \pm 35$ days). It is also apparent that these one-time measurements vary and that babies at the time that they might be operated on (approximately 41 weeks or 287 days) vary in their MAP and weight. It is interesting to note that the MAPs in infants who had similar gestational ages at the time of delivery (GAD) as the ETROP babies also had variable MAPs.

Figure 2 shows the MAP in two patients measured multiple times over a $24 \mathrm{~h}$ period. The patients' GAD were similar to the GAD of retinal patients in ETROP. The MAPs in both patients were labile $(25-56 \mathrm{~mm} \mathrm{Hg})$ during the $24 \mathrm{~h}$ period. All BP measurements were made with a standard cuff, and taken irrespective of the alertness of the baby, the level of stimulation, or feeding schedule.

\section{Discussion}

It seems clear that once the macula is detached due to ROP, the visual results with or without surgery are worse than in stage $4 \mathrm{~A}^{3,7}$ Thus, macular detachment per se is a risk factor for poor vision. It is also clear that scleral buckling for stage $4 \mathrm{~A}$ detachment is better than observation. ${ }^{15,16}$ However, if a vitrectomy is done for $4 \mathrm{~A}$ detachment, only four of eighteen eyes in ETROP had favourable visual acuity and two additional ones had poor but measurable acuity, even though the macula was attached postoperatively in five and some retina was attached in another five. This raises the question whether vitrectomy gives poorer results than scleral buckling for stage $4 \mathrm{~A}$.

In the case of $4 \mathrm{~B}$ detachment, the ETROP numbers are too small to reach conclusions. In stage 5 , no patients had scleral buckling only and none had measurable vision following surgery. Again the question arises whether the presence of macular RD at any time during the immediate post-natal period is the determining factor for vision or whether factors such as inadequate perfusion of the eye before, during, or after vitrectomy could contribute to poor vision.

In ophthalmology we are constantly reminded, especially in the field of glaucoma, that OPP is an important element of good visual function. It is the imbalance between IOP and BP that can lead to glaucomatous damage, which can then lead to optic atrophy. In acute glaucoma especially, where OPP may approach zero, this can happen quickly. ${ }^{17}$ In ROP, poor OPP related to surgery may also cause optic atrophy.

The first question is whether a premature retina needs a greater perfusion pressure than a more mature retina. Also, we do not know, in premature infants with incomplete vascularization of a detached retina, whether there is enough diffusion of nutrients through the subretinal fluid to prevent the retina from degenerating, since the peripheral retina is still avascular. In addition, the remainder of the retina, which appears vascularized, may have a greater nutritive need than an adult retina that may be detached. We do know that RD, if highly detached from the choroid (eg stage 5 ROP) for a prolonged period of time, causes a degeneration and disorientation of photoreceptors, which does not recover well. ${ }^{18}$ In the ROP population, the detachments were low lying in stages $4 \mathrm{~A}$ and $4 \mathrm{~B}$ and highly elevated from the choroid in stage 5, making it more likely that stage 5 ROP would result in poorer visual results. In addition, if the choroidal circulation was compromised, then the chance of visual recovery, even in stage $4 \mathrm{~B}$, would be low, since diffusion of nutrients through the subretinal space would be minimal. 

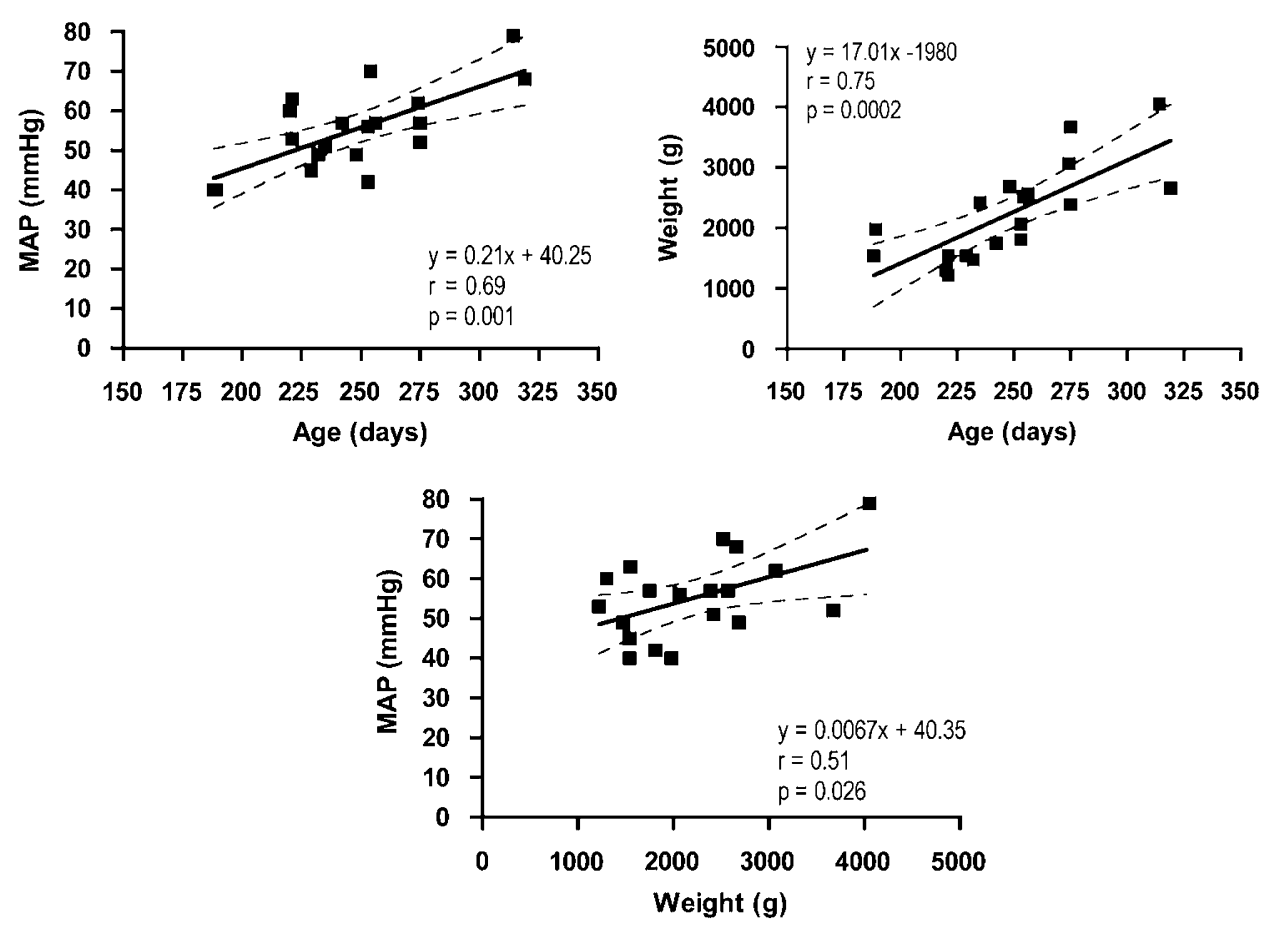

Figure 1 Top left and right: mean arterial pressure (MAP) and body weight (Weight) increase with gestational age (Age). Bottom: MAP increases with weight.

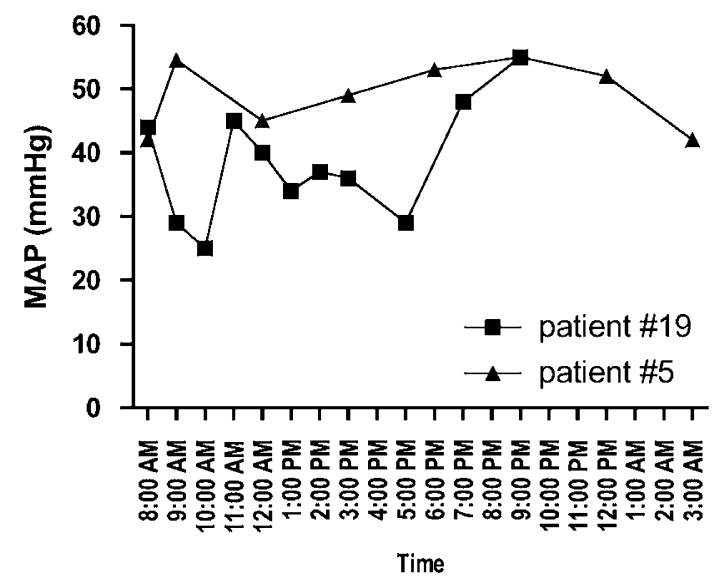

Figure 2 Temporal variation in mean arterial pressure (MAP) in two subjects.

A second question is whether the clinical observation of the presence of a retinal circulation during or after surgery is an adequate measure of the persistence of choroidal blood flow and whether the choroid and retina perfuse in parallel or whether one circulation essentially ceases before the other.

Other questions relate to the influence of our surgical manipulations on OPP and whether operative or post-operative conditions could threaten perfusion.

The first question is difficult to answer. We may never know what the metabolic needs of an immature developing retina are, whether vascularized or not. However, it seems likely that it is not less than that of a mature retina, ${ }^{19}$ so that deprivation by RD (posterior retinal layers separated from their choroidal blood supply) or by a low OPP may be worse for vision in neonates than in older patients.

To answer the second question, we are presently undertaking a study to determine if choroidal and retinal perfusion pressures are similar in other species with retinal and choroidal circulations similar to humans. If similar, then we could be more certain that the operative or post-operative appearance of a patent retinal vasculature is indeed a good and safe measure of the patency of the choroid as well.

In vitrectomy, where infusion is often set at $30 \mathrm{~mm} \mathrm{Hg}$, OPP may reach zero. At times, when intraoperative bleeding occurs, the infusion pressure may be transiently increased above 30 . What the real IOP is during surgery, and for how long, depends on the constantly changing parameters of vitrectomy cutting and suction speed, infusion rate (instrument gauge), as well as the constantly changing MAP, as monitored and controlled by the anaesthesiologist. Also, during vitrectomy, where the surgeon concentrates on the peripheral retina, the 'safety check' of observing the disc circulation may be less often performed, while the choroidal circulation cannot be monitored visually.

Concerning anaesthesia during surgery, anaesthesiologists should be aware that the BP that is 
measured may not be accurate, even with the use of an appropriately sized cuff. Arterial pressures are commonly lower than cuff pressures. The BP reading also depends on the child's position and the delicate balance between sedation and stimulation. Patent ductus status must be known and appropriate drugs must be used which minimize hypotension, and vasopressive drugs as well as cortisol ${ }^{20-22}$ must be available to keep the OPP as high as possible, which requires the anaesthesiologist to know the IOP. If hypotension persists, the surgeon must be asked to lower the IOP to achieve a positive OPP. However, one noteworthy caveat is that systemically administered vasoconstrictors will reach the eye and constrict the arterial vessels supplying the choroid and optic nerve head, so their blood flow may decrease despite the increased OPP; whether the retinal blood vessels also vasoconstrict will depend on the integrity of the immature blood-retinal barrier.

Postoperatively, especially if intraocular air or gas is present in the eye, the IOP may be elevated by numerous mechanisms, including angle closure. Thus, tonometry should be done in the NICU, and the IOP should be kept as low as possible, certainly below the MAP, using glaucoma medications.

To summarize, macular detachment at any time is bad for vision, and it is wise to try to re-attach the macula as soon as possible. However, it seems likely that the physiologic parameters that exist during and after vitrectomy surgery may well compromise the OPP, also leading to retinal and choroidal ischaemia, which may further damage the retina and optic nerve to such an extent that visual results are poor. This is especially true in infants who have the greatest OPP instability, which are those whose birth weight is low and who receive early surgical intervention.

We thus make the following recommendations for infants who have ROP and who are at high risk for or have already developed threshold ROP requiring laser treatment. This is the group of infants from whom a small percentage will develop RD. We suggest that these infants undergo frequent BP measurements in the standard way proposed by Nwankwo. If an arterial line is present, regular direct BP measurements would be advantageous. If the ROP progresses to stage 4 or 5 and vitrectomy is planned, this record must be made available to the ROP surgeon to make them aware of the stability of BP and the lowest BP in any given day. On the day of surgery, these factors must be discussed with the anaesthesiologist so that MAP measured by the anaesthesiologist and IOP controlled by the surgeon are in appropriate balance. Anaesthetic agents must be used to minimize intractable hypotension and appropriate drugs need to be available to increase BP when needed. ${ }^{20-22}$ The surgical and anaesthetic times should be minimized. During surgery, the infusion pressure should be as low as possible and not be routinely set at 30 . The central retinal artery should be observed often. At the end of surgery, the IOP must be taken. Postoperatively, IOP control, using glaucoma drugs, should be used to bring the IOP to $15 \mathrm{~mm} \mathrm{Hg}$ or less. Following surgery, BP should be taken hourly according to a standard protocol as well as frequent IOP measurements, using a pneumotonometer located at the bedside. The patient should not be discharged until BP and IOP are stable and appropriately balanced. If gas or air was used during surgery, infants must be kept prone to avoid angle closure.

In addition, to learn more about the effect of such measures on functional visual outcome, accurate records of BP before, during, and after surgery as well as IOP and other data, as well as information about anaesthetic and glaucoma drugs should be kept for future analysis, much of it in the operative report.

If these suggested measures are taken, it is hoped that the visual results of vitrectomy surgery in this population will improve.

\section{Acknowledgements}

This work was supported by Lew R Wasserman Merit Award (JWK) and an unrestricted departmental grant from Research to Prevent Blindness Inc. and NIH Grant EY 0909702 (JWK).

\section{References}

1 Quinn GE, Dobson V, Barr CC, Davis BR, Flynn JT, Palmer EA et al. Visual acuity in infants after vitrectomy for severe retinopathy of prematurity. Ophthalmology 1991; 98: 5-13.

2 Kiel JW, van Heuven WAJ. Ocular perfusion pressure and choroidal blood flow in the rabbit. Invest Ophthalmol Vis Sci 1995; 36: 579-585.

3 Cryotherapy for Retinopathy of Prematurity Cooperative Group. Multicenter trial of cryotherapy for retinopathy of prematurity: ophthalmological outcomes at 10 years. Arch Ophthalmol 2001; 119: 1110-1118.

4 Good WV. Final results of the Early Treatment for Retinopathy of Prematurity (ETROP) randomized trial. Trans Am Ophthalmol Soc 2004; 102: 233-248;discussion 248-50.

5 International Committee for the Classification of Retinopathy of Prematurity. An international classification of retinopathy of prematurity. Arch Ophthalmol 1984; 102: 1130-1134.

6 International Committee for the Classification of Retinopathy of Prematurity. The international classification of retinopathy of prematurity revisited. Arch Ophthalmol 2005; 123: 991-999.

7 Repka MX, Tung B, Good WV, Shapiro M, Capone Jr A, Baker JD et al. Outcome of eyes developing retinal 
detachment during the Early Treatment for Retinopathy of Prematurity study (ETROP). Arch Ophthalmol 2006; 124: 24-30.

8 Batton DG, Barrington KJ, Wallman C. Prevention and management of pain in the neonate: an update. Pediatrics 2006; 118: 2231-2241.

9 de Swiet M, Fayers P, Shinebourne EA. Systolic blood pressure in a population of infants in the first year of life: the Brompton study. Pediatrics 1980; 65: 1028-1035.

10 Nwankwo MU, Lorenz JM, Gardiner JC. A standard protocol for blood pressure measurement in the newborn. Pediatrics 1997; 99: E10.

11 Victor S, Marson AG, Appleton RE, Beirne M, Weindling AM. Relationship between blood pressure, cerebral electrical activity, cerebral fractional oxygen extraction, and peripheral blood flow in very low birth weight newborn infants. Pediatr Res 2006; 59: 314-319.

12 Short BL, Van Meurs K, Evans JR. Summary proceedings from the cardiology group on cardiovascular instability in preterm infants. Pediatrics 2006; 117: S34-S39.

13 Evans JR, Lou Short B, Van Meurs K, Cheryl Sachs H. Cardiovascular support in preterm infants. Clin Ther 2006; 28: 1366-1384.

14 Dempsey EM, Barrington KJ. Diagnostic criteria and therapeutic interventions for the hypotensive very low birth weight infant. J Perinatol 2006; 26: 677-681.
15 Capone Jr A, Trese MT. Lens-sparing vitreous surgery for tractional stage $4 \mathrm{~A}$ retinopathy of prematurity retinal detachments. Ophthalmology 2001; 108: 2068-2070.

16 Hubbard III GB, Cherwick DH, Burian G. Lens-sparing vitrectomy for stage 4 retinopathy of prematurity. Ophthalmology 2004; 111: 2274-2277.

17 Hayreh SS, Zimmerman MB, Kimura A, Sanon A. Central retinal artery occlusion. Retinal survival time. Exp Eye Res 2004; 78: 723-736.

18 Kroll AJ, Machemer R. Experimental retinal detachment in the owl monkey. V. Electron microscopy of the reattached retina. Am J Ophthalmol 1969; 67: 117-130.

19 Fanaroff AA, Fanaroff JM. Short- and long-term consequences of hypotension in ELBW infants. Semin Perinatol 2006; 30: 151-155.

20 Ng PC, Lee CH, Bnur FL, Chan IH, Lee AW, Wong E et al. A double-blind, randomized, controlled study of a 'stress dose' of hydrocortisone for rescue treatment of refractory hypotension in preterm infants. Pediatrics 2006; 117: 367-375.

21 Seri I, Tan R, Evans J. Cardiovascular effects of hydrocortisone in preterm infants with pressor-resistant hypotension. Pediatrics 2001; 107: 1070-1074.

22 Noori S, Friedlich P, Wong P, Ebrahimi M, Siassi B, Seri I. Hemodynamic changes after low-dosage hydrocortisone administration in vasopressor-treated preterm and term neonates. Pediatrics 2006; 118: 1456-1466. 